\title{
Figure of Merit and Different Combinations of Observational Data Sets
}

\author{
Qiping $\mathrm{Su}^{1,2} *$ Zhong-Liang $\mathrm{Tuo}^{2}+\dagger$ and Rong-Gen Cai2 ${ }^{+}$ \\ 1 Department of Physics, Hangzhou Normal University, Hangzhou, 310036, China \\ 2 Key Laboratory of Frontiers in Theoretical Physics, \\ Institute of Theoretical Physics, Chinese Academy of Sciences, \\ P.O. Box 2735, Beijing 100190, China
}

(Dated: October 31, 2018)

\begin{abstract}
To constrain cosmological parameters, one often makes a joint analysis with different combinations of observational data sets. In this paper we take the figure of merit (FoM) for Dark Energy Task Force fiducial model (CPL model) to estimate goodness of different combinations of data sets, which include 11 widely-used observational data sets (Type Ia Supernovae, Observational Hubble Parameter, Baryon Acoustic Oscillation, Cosmic Microwave Background, X-ray Cluster Baryon Mass Fraction, and Gamma-Ray Bursts). We analyze different combinations and make a comparison for two types of combination based on two types of basic combinations, which are often adopted in the literatures. We find two sets of combinations, which have strong ability to constrain the dark energy parameters, one has the largest FoM, the other contains less observational data with a relative large FoM and a simple fitting procedure.
\end{abstract}

\footnotetext{
*Electronic address: sqp@hznu.edu.cn

${ }^{\dagger}$ Electronic address: tuozhl@itp.ac.cn

${ }^{\ddagger}$ Electronic address: cairg@itp.ac.cn
} 


\section{INTRODUCTION}

Since the discovery of cosmic acceleration expansion of the universe [1, 2], its origin has been a hot topic in modern cosmology and theoretical physics. Various dark energy (DE) models explaining the acceleration expansion have been proposed [3-14], which originate from extra dimensions, string theory, supergravity, extended standard model of particle physics, and so on. Most of the DE models can fit astronomical observational data. At present, the main task of DE studies is to constrain the present equation of state (EoS) of $\mathrm{DE} w_{d e 0}$, the fractional energy density $\Omega_{d e 0}$ and to give the possible evolution form of $w_{d e}(z)$. Several parameterization forms of $w_{d e}(z)$ have been proposed, such as the CPL parameterization $w_{d e}(z)=w_{0}+w_{1} z /(1+z)$ [15, 16] and the parameterization $w_{d e}(z)=$

$w_{0}+w_{1} z[17-20]$. Fitting the parameterization models of DE with present observational data, constraints of DE can be obtained. The fitting results favor that the present value of $w_{d e}$ is very close to -1 and its variation is very slow. But it still not be able to confirm whether $w_{d e}$ is evolving or just a constant. Of course, these constraints are also dependent on the parameterization forms used. As a result several methods have been proposed to get model-independent constraints of DE from observations [21 27], such as the uncorrelated bandpower estimate (UBE) [28] and the spline method [29 31].

On the other hand, the fitting results also depend on the observational data used and there might exist "tensions" among the estimated EoS parameters from different data sets [32 38]. A single observation still gives poor constraints on the parameters of cosmological models. For example, Type Ia supernovae observation, which has large number of datapoints, gives poor constraints on $\Omega_{m 0}$ and $w_{d e}$ because of the degeneracy between $\Omega_{m 0}$ and $w_{d e}[39]$. To break the degeneracy and get good constraints of the parameters, one always combines different types of observation to fit cosmological models. It is not surprising that fitting a DE model with different combinations of observational data sets, one would get different constraints on DE. There seemingly does not exist a general rule to combine different observations for fitting the cosmological parameters. One uses different combinations in different works.

In this paper we would like to make a comparison for different combinations of widelyused data sets for DE, which contain data of Type Ia Supernovae (SN), Observational Hubble Parameter (HUB), Baryon Acoustic Oscillation (BAO), Cosmic Microwave Back- 
ground (CMB), X-ray Cluster Baryon Mass Fraction (CBMF), Gamma-Ray Bursts (GRB), etc. The fitting properties of observational data will be studied by using the Dark Energy Task Force (DETF) [40-42] fiducial model (CPL model), i.e., all combinations of data will be fitted with the CPL parameterization. The best-fitted values of $\Omega_{m 0}, w_{0}, w_{1}$ and their $68 \%, 95 \%$ C.L. errors for each combination will be given. To estimate constraints on $w_{d e}$ and compare the goodness of data, we will calculate the figure of merit (FoM) [40-42] for each combination, which is proportional to the inverse area of the error ellipse in the $w_{0} \sim w_{1}$ plane. Namely we will take the FoM as the diagnostic to quantify the goodness of each combination of observational data to constrain the DE parameters.

The paper is organized as follows. In Section II, we introduce 11 sets of observational data and study their fitting properties on the basis of Type Ia supernovae Union2 data [43]. In Section III we study the effect of different combinations of data sets on constraining DE parameters. We divide the combinations into two types, based on two basic combinations, $\mathrm{SN}+\mathrm{BAOI}+\mathrm{CMB}$ and $\mathrm{SN}+\mathrm{A}+\mathrm{R}$, respectively. The properties of various combinations will be analyzed and compared, and the best combination (which has the largest FoM) will be given. Main results are summarized in Section IV.

\section{OBSERVATIONAL DATA SETS}

One of important astronomical observations to constrain DE is the distance measurement (such as SN and shift parameter R). To constrain DE models with these data, one must give the form of Hubble function $H(z)$ (or $E(z)=H(z) / H_{0}$ ). In a flat Friedmann-RobertsonWalker (FRW) universe, one has

$$
E^{2}(z)=\Omega_{r 0}(1+z)^{4}+\Omega_{b 0}(1+z)^{3}+\Omega_{d m 0}(1+z)^{3}+\Omega_{d e 0} F(z),
$$

where $\Omega_{r 0}, \Omega_{b 0}, \Omega_{d m 0}$ and $\Omega_{d e 0}$ are present values of the fractional energy density for radiations, baryons, dark matter and dark energy respectively, with $\Omega_{r 0}+\Omega_{b 0}+\Omega_{d m 0}+\Omega_{d e 0}=1$. The energy densities of baryons and dark matter are often written together as $\Omega_{b 0}+\Omega_{d m 0}=$ $\Omega_{m 0}$. The radiation density is the sum of photons and relativistic neutrinos [44]:

$$
\Omega_{r}^{(0)}=\Omega_{\gamma}^{(0)}\left(1+0.2271 N_{n}\right),
$$

where $N_{n}$ is the number of neutrino species and $\Omega_{\gamma}^{(0)}=2.469 \times 10^{-5} h^{-2}$ for $T_{c m b}=2.725 \mathrm{~K}$ $\left(h=H_{0} / 100 \mathrm{Mpc} \cdot \mathrm{km} \cdot \mathrm{s}^{-1}\right)$. The evolving function $F(z)$ for DE depends on the equation 
of state, $w_{d e}(z)$, of DE models,

$$
F(z)=e^{3 \int_{0}^{z} \frac{1+w_{d e}}{1+x} d x}
$$

We will fit observational data with the CPL parameterization. In that case, one has

$$
F(z)=(1+z)^{3\left(1+w_{0}+w_{1}\right)} \exp \left[-\frac{3 w_{1} z}{1+z}\right] .
$$

When fitting the CPL model with data of distance measurement, the parameters $\Omega_{m 0}$ (or $\left.\Omega_{d e 0}\right), w_{0}, w_{1}$, and $h$ are needed. While for some other data, such as those of CMB and BAO, one needs to calculate physical quantities other than the distance (such as the redshift of decoupling, $z_{s}$, and the comoving sound horizon at decoupling, $r_{s}$ ) and the parameter $\Omega_{b 0}$ must be involved. Whenever the fitting procedure needs to use $\Omega_{b 0}$, one more datapoint will always be adopted to constrain $\Omega_{b 0}$ and $h[45]$ :

$$
\Omega_{b 0} h^{2}=0.022 \pm 0.002
$$

In addition, in all calculations we will assume the prior that the age of the universe $T_{0}$ satisfies 10 Gyr $<T_{0}<20$ Gyr.

We will use Union2 SN data [43] and other widely-used 10 observational data sets to constrain the CPL model. When studying the fitting properties of these 10 observational data, we will combine each data set with the SN data to fit CPL model. Since we are only interested in the constraints on DE, only the best-fitted values of $\Omega_{m 0}, w_{0}, w_{1}$ and their $68 \%$, 95\% C.L. errors will be given, which are obtained by using the Markov Chain Monte Carlo method. To estimate and compare the goodness of constraints from the observational data sets, we calculate the figure of merit (FoM) [40 42, 46] for each combination of data sets, which is proportional to the inverse area of the error ellipse in the $w_{0} \sim w_{1}$ plane:

$$
\mathrm{FoM}=\left[\operatorname{det} C\left(w_{0}, w_{1}\right)\right]^{-1 / 2}
$$

where $C\left(w_{0}, w_{1}\right)$ is the covariance matrix of $w_{0}$ and $w_{1}$ after marginalizing out all of the other cosmological parameters. Larger FoM means stronger constraint on the parameters since it corresponds to a smaller error ellipse.

\section{A. Type Ia Supernovae (SN)}

Type Ia supernovae observation gives the direct evidence for the existence of the acceler-

ation expansion of the universe. In this work we take the Union2 SN dataset [43], for which 


\begin{tabular}{|c|c|c|c|c|c|}
\hline data & $\Omega_{m 0}$ & $w_{0}$ & $w_{1}$ & $\chi_{\text {min }}^{2}$ & FoM \\
\hline \hline $\mathrm{SN}$ & $0.419_{-0.028-0.238}^{+0.090+0.133}$ & $-0.86_{-0.32-0.59}^{+0.46+1.19}$ & $-5.51_{-8.79-2.4 .09}^{+6.99+7.59}$ & 541.431 & 0.647 \\
\hline $\mathrm{SN}+\mathrm{GRB}$ & $0.413_{-0.035-0.310}^{+0.091+0.132}$ & $-0.88_{-0.30-0.54}^{+0.42+1.16}$ & $-5.02_{-7.52-19.86}^{+6.48+7.13}$ & 564.727 & 0.700 \\
\hline $\mathrm{SN}+\mathrm{CBMF}$ & $0.378_{-0.025-0.097}^{+0.085+0.126}$ & $-0.97_{-0.23-0.47}^{+0.31+0.71}$ & $-2.77_{-4.90-13.09}^{+2.93+4.36}$ & 583.725 & 1.356 \\
\hline $\mathrm{SN}+\mathrm{HUB}$ & $0.281_{-0.027-0.217}^{+0.134+0.170}$ & $-1.00_{-0.12-0.34}^{+0.22+0.47}$ & $-0.21_{-2.82-8.46}^{+1.54+1.98}$ & 550.391 & 2.315 \\
\hline $\mathrm{SN}+\mathrm{CMB}$ & $0.274_{-0.017-0.036}^{+0.025+0.051}$ & $-1.01_{-0.14-0.34}^{+0.16+0.2}$ & $0.01_{-0.84-1.54}^{+0.73+1.45}$ & 542.697 & 21.688 \\
\hline $\mathrm{SN}+\mathrm{R}$ & $0.277_{-0.021-0.040}^{+0.021+0.045}$ & $-1.01_{-0.14-0.30}^{+0.17+0.31}$ & $-0.06_{-0.84-1.64}^{+0.75+1.38}$ & 542.642 & 21.376 \\
\hline
\end{tabular}

TABLE I: The best-fitted values with $68 \%$ and $95 \%$ C.L. errors of $\Omega_{m 0}, w_{0}, w_{1}$, and figure of merit for different combinations of data sets in the CPL model.

$\chi_{S N}^{2}$ is given by

$$
\chi_{S N}^{2}=\sum_{i=1}^{557} \frac{\left[\mu_{t h}\left(z_{i}\right)-\mu_{o b}\left(z_{i}\right)\right]^{2}}{\sigma^{2}\left(z_{i}\right)},
$$

where the theoretical distance modulus $\mu_{t h}$ is defined as

$$
\mu_{t h}(z)=5 \log _{10} D_{L}+\mu_{0}, \quad \mu_{0}=42.384-5 \log _{10} h
$$

where the luminosity distance $D_{L}=(1+z) \int_{0}^{z} d x / E(x)$. One can expand Eq.(7) with respect to $\mu_{0}$ as

$$
\chi_{S N}^{2}=\tilde{A}+2 \tilde{B} \mu_{0}+\tilde{C} \mu_{0}^{2}
$$

where

$$
\begin{aligned}
& \tilde{A}=\sum_{i=1}^{557} \frac{\left[\mu_{t h}\left(z_{i} ; \mu_{0}=0\right)-\mu_{o b}\left(z_{i}\right)\right]^{2}}{\sigma^{2}\left(z_{i}\right)} \\
& \tilde{B}=\sum_{i=1}^{557} \frac{\mu_{t h}\left(z_{i} ; \mu_{0}=0\right)-\mu_{o b}\left(z_{i}\right)}{\sigma^{2}\left(z_{i}\right)} \\
& \tilde{C}=\sum_{i=1}^{557} \frac{1}{\sigma^{2}\left(z_{i}\right)}
\end{aligned}
$$

The $\chi_{S N}^{2}$ has a minimum as

$$
\tilde{\chi}_{S N}^{2}=\tilde{A}-\tilde{B}^{2} / \tilde{C}
$$

We will use $\tilde{\chi}_{S N}^{2}$ instead of $\chi_{S N}^{2}$, this way the nuisance parameter $\mu_{0}$ is reduced. It is equivalent to performing an uniform marginalization over $\mu_{0}$.

In Table II it shows that the SN data give a largish $\Omega_{m 0}$ and the value of FoM is rather small, which means that the constraint on the parameters is weak. This is mainly due to the 
degeneracy between $\Omega_{m 0}$ and $w_{d e}$. Thus the SN data are often combined with other types of observations, especially that can constrain $\Omega_{m 0}$ tightly, such as BAO and CMB.

\section{B. Gamma-Ray Bursts (GRB)}

Gamma-Ray Bursts (GRB) are the most intense explosions we have observed in the universe, most GRB observed are in the range $0.1<z<8.1$. Thus, GRB are considered to be a complementary probe to SN and a hopeful probe at high redshift. Here we adopt the 59 Hymnium GRB at $z>1.4$ from [47], which are obtained by calibrating the 109 GRB with Amati relation, using the cosmological independent method proposed in [48].

The operation of GRB datapoints is the same as that of SN. To fit those data with DE models it should be combined with $\mathrm{SN}$ data, i.e., $\chi_{G R B}^{2}$ should be calculated together with $\chi_{S N}^{2}$ as

$$
\tilde{\chi}_{S N+G R B}^{2}=\bar{A}-\bar{B}^{2} / \bar{C}
$$

where

$$
\begin{aligned}
& \bar{A}=\sum_{j=1}^{557+59} \frac{\left[\mu_{t h}\left(z_{j} ; \mu_{0}=0\right)-\mu_{o b}\left(z_{j}\right)\right]^{2}}{\sigma^{2}\left(z_{j}\right)}, \\
& \bar{B}=\sum_{j=1}^{557+59} \frac{\mu_{t h}\left(z_{j} ; \mu_{0}=0\right)-\mu_{o b}\left(z_{j}\right)}{\sigma^{2}\left(z_{j}\right)}, \\
& \bar{C}=\sum_{j=1}^{557+59} \frac{1}{\sigma^{2}\left(z_{j}\right)} .
\end{aligned}
$$

The datapoints in the summation contain $557 \mathrm{SN}$ and 59 GRB.

The results for SN+GRB are shown in Table I, which are almost the same as that of SN. With addition of $59 \mathrm{GRB}$ data, the increase of $\chi^{2}$ (about 23) is much less than the increase of number of datapoints, which implies that the present precision of the GRB observation is not small enough. As a result it means that adding GRB data to SN data is not quite helpful to constrain the DE model.

\section{X-ray Cluster Baryon Mass Fraction (CBMF)}

The baryon mass fraction in clusters of galaxies (CBMF) are also used to constrain

cosmological parameters. Here we adopt the updated 42 observational $f_{\text {gas }}$ data in [49]. 
The X-ray gas mass fraction is defined as the ratio of the X-ray gas mass to the total mass of a cluster, which should be a redshift-independent constant. With the $\Lambda \mathrm{CDM}$ reference cosmological model, one has

$$
f_{g a s}(z)=\frac{K \gamma b}{1+s}\left(\frac{\Omega_{b}}{\Omega_{m}}\right) A\left[\frac{D_{A}^{\Lambda C D M}(z)}{D_{A}(z)}\right]^{3 / 2},
$$

where $A$ is the angular correction factor, which is due to the change in angle subtended by $r_{2500}$ as the underlying cosmological model is changed:

$$
A=\left(\frac{\theta_{2500}^{\Lambda C D M}}{\theta_{2500}}\right)^{\eta} \approx\left(\frac{H(z) D_{A}(z)}{\left[H(z) D_{A}(z)\right]^{\Lambda C D M}}\right)^{\eta} .
$$

Here we adopt the best-fitted average value $\eta=0.214$, and the proper angular diameter distance is given by

$$
D_{A}(z)=\frac{D_{L}(z)}{(1+z)^{2}} .
$$

For other parameters K, $\gamma, b, s$ in Eq.(13), please refer to Refs. [49, 50]. Here we introduce a new parameter $\lambda[51]$ :

$$
\lambda \equiv \frac{K \gamma b}{1+s}\left(\frac{\Omega_{b}}{\Omega_{m}}\right),
$$

then one has

$$
f_{\text {gas }}\left(z_{i}\right)=\lambda A\left[\frac{D_{A}^{\Lambda C D M}\left(z_{i}\right)}{D_{A}\left(z_{i}\right)}\right]^{3 / 2} \equiv \lambda \widehat{f}_{\text {gas }}\left(z_{i}\right) .
$$

Next one can expand

$$
\chi_{C B M F}^{2} \equiv \sum_{i} \frac{\left(f_{g a s}\left(z_{i}\right)-f_{g a s, i}^{o b s}\right)^{2}}{\sigma_{i}^{2}}
$$

with respect to $\lambda$ and obtain

$$
\chi_{C B M F}^{2}=\widehat{A} \lambda^{2}-2 \widehat{B} \lambda+\widehat{C},
$$

where

$$
\begin{aligned}
\widehat{A} & =\sum_{i} \frac{\widehat{f}_{\text {gas }}\left(z_{i}\right)^{2}}{\sigma_{i}^{2}}, \\
\widehat{B} & =\sum_{i} \frac{\widehat{f}_{\text {gas }}\left(z_{i}\right) f_{\text {gas }, i},}{\sigma_{i}^{2}}, \\
\widehat{C} & =\sum_{i} \frac{\left(f_{\text {gas }, i}^{o b s}\right)^{2}}{\sigma_{i}^{2}} .
\end{aligned}
$$

As in the case of SN, instead of $\chi_{C B M F}^{2}$, here we adopt the minimum of $\chi_{C B M F}^{2}$ with respect to $\lambda$,

$$
\widehat{\chi}_{C B M F}^{2}=\widehat{C}-\widehat{B}^{2} / \widehat{A},
$$


as $\chi^{2}$ for CBMF.

As shown in Table I, the constraint from SN+CBMF is also weak, but better than that of $\mathrm{SN}+\mathrm{GRB}$. The best fitted value of $\Omega_{m 0}$ is still largish. The addition of $42 \mathrm{CBMF}$ to SN data does not improve much.

\section{Observational Hubble Data (HUB)}

The observational Hubble data can be obtained by using the differential ages of passivelyevolving galaxies as

$$
H \simeq-\frac{1}{1+z} \frac{\Delta z}{\Delta t}
$$

We use 12 observational Hubble data from [52] and [53], which is tabled in [50]. The chisquare is defined as:

$$
\chi_{H U B}^{2}=\sum_{i=1}^{12} \frac{\left[H_{t h}\left(z_{i}\right)-H_{o b}\left(z_{i}\right)\right]^{2}}{\sigma_{i}^{2}} .
$$

In this case we use these values of $H\left(z_{i}\right)$ directly, which can break the degeneracy between $\Omega_{m 0}$ and $w_{d e}$. With these data, as shown in Table I, there is an increase of FoM and the best-fitted $\Omega_{m 0}$ becomes much smaller than in the case of the SN data used only.

\section{E. Cosmic Microwave Background (CMB)}

\section{1. $\mathrm{CMB}$}

In the CMB measurement, the distance to the decoupling epoch can be accurately determined from the locations of peaks and troughs of acoustic oscillations. For simplicity, we use three parameters $l_{a}, R, z_{s}$ obtained from WMAP7 (rather than the full data of WMAP) [54] to constrain cosmological models. Here $z_{s}$ is the redshift of decoupling [55], the shift parameter $R$ is the scaled distance to the decoupling epoch:

$$
R=\sqrt{\Omega_{m 0}} \int_{0}^{z_{s}} \frac{d z}{E(z)}
$$

and $l_{a}$ is the angular scale of the sound horizon at the decoupling epoch:

$$
l_{a}=\pi \frac{r\left(a_{s}\right)}{r_{s}\left(a_{s}\right)}
$$


where $r(z)=\int_{0}^{z} d x / H(x)$ is the comoving distance and $r_{s}\left(a_{s}\right)$ is the comoving sound horizon at the decoupling epoch:

$$
r_{s}\left(a_{s}\right)=\int_{0}^{a_{s}} \frac{c_{s}(a)}{a^{2} H(a)} d a, \quad a_{s}=\frac{1}{1+z_{s}},
$$

where the sound speed $c_{s}(a)=1 / \sqrt{3\left(1+\bar{R}_{b} a\right)}$ and $\bar{R}_{b}=3 \Omega_{b 0} / 4 \Omega_{\gamma 0}$ is the photon-baryon energy density ratio.

The $\chi^{2}$ of the CMB data is constructed as:

$$
\chi_{C M B}^{2}=X^{T} C_{M}^{-1} X
$$

where

$$
X=\left(\begin{array}{c}
l_{a}-302.09 \\
R-1.725 \\
z_{s}-1091.3
\end{array}\right)
$$

and the inverse covariance matrix

$$
C_{M}^{-1}=\left(\begin{array}{ccc}
2.305 & 29.698 & -1.333 \\
29.698 & 6825.270 & -113.180 \\
-1.333 & -113.180 & 3.414
\end{array}\right)
$$

In this case the parameter $\Omega_{b 0}$ is involved. From the definition of the shift parameter $R$ [see (24)], it is obvious that $\Omega_{m 0}$ can be well constrained, and the degeneracy between $\Omega_{m 0}$ and $w_{d e}$ could be broken in some sense. As shown in Table I, the FoM and constraints of parameters have a great improvement from those in the case of SN data used only. Therefore the CMB data are a good supplementary of SN data in constraining DE models.

2. Shift Parameter $R$

As an alternative, one can only adopt the shift parameter $R$ from WMAP7 [54] to constrain the cosmological parameters. In this case the corresponding $\chi^{2}$ is defined as

$$
\chi_{R}^{2}=\left(\frac{R-1.725}{0.018}\right)^{2}
$$

It can be seen from Table 1 that the fitting results of $\mathrm{SN}+\mathrm{R}$ are very close to those of $\mathrm{SN}+\mathrm{R}+l_{a}+z_{s}$ (i.e., $\left.\mathrm{SN}+\mathrm{CMB}\right)$. Note that in this case one needs not to handle with $\Omega_{b 0}$ and the calculation is much simpler. The addition of a single datapoint $R$ to SN data greatly improves the fitting results, because $R$ can alleviate the degeneracy between $\Omega_{m 0}$ and $w_{d e}$ well. 


\begin{tabular}{|c|c|c|c|c|c|}
\hline data & $\Omega_{m 0}$ & $w_{0}$ & $w_{1}$ & $\chi_{\text {min }}^{2}$ & FoM \\
\hline \hline $\mathrm{SN}+\mathrm{BAOI}$ & $0.420_{-0.066-0.177}^{+0.036+0.068}$ & $-0.84_{-0.32-0.53}^{+0.23+0.71}$ & $-5.79_{-2.60-10.25}^{+6.65+7.06}$ & 542.186 & 1.471 \\
\hline $\mathrm{SN}+\mathrm{BAOII}$ & $0.428_{-0.033-0.359}^{+0.083+0.122}$ & $-0.80_{-0.35-0.59}^{+0.46+1.20}$ & $-6.50_{-8.78-26.82}^{+7.77+8.45}$ & 542.643 & 0.632 \\
\hline $\mathrm{SN}+\mathrm{BAOIII}$ & $0.421_{-0.066-0.190}^{+0.035+0.066}$ & $-0.82_{-0.33-0.55}^{+0.23+0.67}$ & $-5.89_{-2.14-8.69}^{+6.73+7.13}$ & 542.134 & 1.514 \\
\hline $\mathrm{SN}+\mathrm{A}$ & $0.278_{-0.018-0.037}^{+0.025+0.047}$ & $-1.01_{-0.13-0.28}^{+0.17+0.33}$ & $-0.10_{-1.27-2.57}^{+1.04+1.89}$ & 542.643 & 13.223 \\
\hline $\mathrm{SN}+\mathrm{RBAO}$ & $0.394_{-0.091-0.241}^{+0.026+0.056}$ & $-0.93_{-0.21-0.41}^{+0.18+0.48}$ & $-3.78_{-0.038-4.35}^{+4.75+5.05}$ & 541.602 & 2.586 \\
\hline
\end{tabular}

TABLE II: The best-fitted values with $68 \%$ and $95 \%$ C.L. errors of $\Omega_{m 0}, w_{0}, w_{1}$, and figure of merit for different combinations of data sets in the CPL model.

\section{F. Baryon Acoustic Oscillation (BAO)}

As baryons and photons are tightly coupled at early times, gravity and pressure gradients induce an acoustic oscillation in the baryon-photon fluid. Since the baryonic matter interacts gravitationally with the dark matter, the acoustic oscillations leaves some fingerprint in the matter power spectrum. The BAO peak length scale is set by the sound horizon at decoupling, $\sim 10^{2} \mathrm{Mpc}$. We now introduce several types of BAO data.

\section{BAOI}

The first one is the BAO distance measurements obtained at $\mathrm{z}=0.2$ and $\mathrm{z}=0.35$ from joint analysis of the 2dFGRS and SDSS DR7 data [56]:

$$
\begin{aligned}
\frac{r_{s}\left(z_{d}\right)}{D_{V}(0.2)} & =0.1905 \pm 0.0061 \\
\frac{r_{s}\left(z_{d}\right)}{D_{V}(0.35)} & =0.1097 \pm 0.0036
\end{aligned}
$$

where $r_{s}\left(z_{d}\right)$ is the comoving sound horizon at the baryon drag epoch $z_{d}$ [57], and

$$
D_{V}(z)=\left[\left(\int_{0}^{z} \frac{d x}{H(x)}\right)^{2} \frac{z}{H(z)}\right]^{1 / 3}
$$

encodes the visual distortion of a spherical object due to the non Euclidianity of a FRW spacetime. The $\chi_{B A O I}^{2}$ is given by

$$
\chi_{B A O I}^{2}=X^{T} V^{-1} X
$$

where

$$
X=\left(\begin{array}{c}
\frac{r_{s}\left(z_{d}\right)}{D_{V}(0.2)}-0.1905 \\
\frac{r_{s}\left(z_{d}\right)}{D_{V}(0.35)}-0.1097
\end{array}\right)
$$


and the inverse covariance matrix

$$
V^{-1}=\left(\begin{array}{cc}
30124.1 & -17226.9 \\
-17226.9 & 86976.6
\end{array}\right)
$$

With addition of the BAOI data to SN data, there is a bit improvement of FoM, as shown

in Table II. The best-fitted parameters are also very close to those from the case of SN data used only. This implies that BAOI data cannot alleviate the degeneracy between $\Omega_{m 0}$ and $w_{d e}$.

\section{BAOII}

Using two distance measurements of BAOI, one can derive a model independent BAO distance ratio [56]

$$
\frac{D_{V}(0.35)}{D_{V}(0.2)}=1.736 \pm 0.065
$$

which is independent of $\Omega_{b 0}$. For this single datapoint, one defines

$$
\chi_{B A O I I}^{2}=\left(\frac{D_{V}(0.35) / D_{V}(0.2)-1.736}{0.065}\right)^{2} .
$$

The FoM of $\mathrm{SN}+\mathrm{BAOII}$ is even smaller than that of $\mathrm{SN}$ data, and the $\chi_{\min }^{2}$ is larger than that of $\mathrm{SN}+\mathrm{BAOI}$ data.

\section{BAOIII}

In this case we combine the data of BAOII with the BAO distance measurements at $z=0.275[56]$ :

$$
\frac{r_{s}\left(z_{d}\right)}{D_{V}(0.275)}=0.1390 \pm 0.0037
$$

These two datapoints have been widely-used in the literatures. In this case the $\chi^{2}$ is defined as

$$
\chi_{\text {BAOIII }}^{2}=\left(\frac{D_{V}(0.35) / D_{V}(0.2)-1.736}{0.065}\right)^{2}+\left(\frac{r_{s}\left(z_{d}\right) / D_{V}(0.275)-0.1390}{0.0037}\right)^{2} .
$$

As shown in Table II, the results for the combination SN+BAOIII are almost the same as those of SN+BAOI.

\section{Distance Parameter $A$}

The distance parameter $A$ is often used in the literatures, which is the measurement of BAO peak in the distribution of SDSS luminous red galaxies [58]:

$$
A=\Omega_{m}^{1 / 2} E(0.35)^{-1 / 3}\left[\frac{1}{0.35} \int_{0}^{0.35} \frac{d z}{E(z)}\right]^{2 / 3}
$$


This quantity is independent of $\Omega_{b 0}$. The value of $A$ is determined to be $0.469\left(n_{s} / 0.98\right)^{-0.35} \pm$ 0.017 , where $n_{s}=0.963$ is the scalar spectral index, which has been updated from the WMAP7 data. $\chi^{2}$ is defined as

$$
\chi_{A}^{2}=\frac{(A-0.472)^{2}}{0.017^{2}} .
$$

The supplementary of the single datapoint $A$ to SN data gives a large increase of FoM, because $\Omega_{m 0}$ can be well constrained and the degeneracy between $\Omega_{m 0}$ and $w_{d e}$ is alleviated.

5. RBAO

SDSS data can also be used to measure the radial (line-of-sight) baryon acoustic scale, which is independent from the previous $\mathrm{BAO}$ measurements which were averaged over all directions or in the transverse direction. In that case the measured quantities are the values of

$$
\Delta_{z}(z)=H(z) r_{s}\left(z_{d}\right)
$$

at $z=0.24$ and $z=0.43[59]$, respectively,

$$
\Delta_{z}(0.24)=0.0407 \pm 0.0011 \pm 0.0007, \quad \Delta_{z}(0.43)=0.0442 \pm 0.0015 \pm 0.0009
$$

with statistic errors and systematic errors. In this case, the parameter $\Omega_{b 0}$ is involved once again.

Fitting results show that obviously this set of data has poor constraints on $\Omega_{m 0}$ and gives poor improvement of FoM, as shown in Table II.

\section{COMBINATIONS OF DATA SETS}

In general, to get good constraints of DE parameters one often combines several types of observational data sets to fit cosmological models. In this section we study the effect of different combinations of the data sets introduced in the previous section on constraining DE models. We divide the combinations of data sets into two types:

Type I: based on $\mathrm{SN}+\mathrm{BAOI}+\mathrm{CMB}$, where the parameter $\Omega_{b 0}$ is involved.

Type II: based on $\mathrm{SN}+\mathrm{A}+\mathrm{R}$, which only depends on the distance measurement. The calculation is much simpler than the case of Type I.

The basic combinations $\mathrm{SN}+\mathrm{BAOI}+\mathrm{CMB}\left(B_{I}\right)$ and $\mathrm{SN}+\mathrm{A}+\mathrm{R}\left(B_{I I}\right)$ are two widely-used combinations in the literatures. The data sets to be combined with two basic combinations 


\begin{tabular}{|c|c|c|c|c|c|}
\hline data & $\Omega_{m 0}$ & $w_{0}$ & $w_{1}$ & $\chi_{\text {min }}^{2}$ & FoM \\
\hline \hline $\mathrm{SN}+\mathrm{BAOI}+\mathrm{CMB}$ & $0.279_{-0.010-0.023}^{+0.017+0.033}$ & $-1.07_{-0.09-0.19}^{+0.14+0.27}$ & $0.28_{-0.76-1.66}^{+0.46+0.84}$ & 544.131 & 27.721 \\
\hline $\mathrm{SN}+\mathrm{BAOI}+\mathrm{CMB}+\mathrm{GRB}$ & $0.281_{-0.012-0.025}^{+0.016+0.031}$ & $-1.07_{-0.08-0.19}^{+0.14+0.28}$ & $0.29_{-0.78-1.74}^{+0.45+0.82}$ & 567.309 & 27.401 \\
\hline $\mathrm{SN}+\mathrm{BAOI}+\mathrm{CMB}+\mathrm{HUB}$ & $0.275_{-0.010-0.023}^{+0.017+0.030}$ & $-1.03_{-0.09-0.19}^{+0.13+0.25}$ & $0.02_{-0.64-1.49}^{+0.52+0.91}$ & 554.034 & 29.495 \\
\hline $\mathrm{SN}+\mathrm{BAOI}+\mathrm{CMB}+\mathrm{RBAO}$ & $0.274_{-0.009-0.019}^{+0.016+0.029}$ & $-1.02_{-0.0-_{-0.18}^{+0.12}}^{+0.24}$ & $0.05_{-0.71-1.52}^{+0.48+0.82}$ & 544.721 & 32.455 \\
\hline $\mathrm{SN}+\mathrm{BAOI}+\mathrm{CMB}+\mathrm{CBMF}$ & $0.279_{-0.009-0.022}^{+0.019+0.034}$ & $-1.07_{-0.09-0.20}^{+0.15+0.28}$ & $0.23_{-0.93-1.76}^{+0.51+0.90}$ & 587.345 & 26.319 \\
\hline $\mathrm{SN}+\mathrm{BAOI}+\mathrm{CMB}+\mathrm{CBMF}+\mathrm{RBAO}$ & $0.278_{-0.012-0.023}^{+0.013+0.025}$ & $-1.03_{-0.08_{-0.17}^{+0.12+0.26}}^{+0.26}$ & $-0.03_{-0.70-1.57}^{+0.52+0.93}$ & 587.990 & 30.803 \\
\hline $\mathrm{SN}+\mathrm{BAOI+CMB+HUB+RBAO}$ & $0.273_{-0.009-0.020}^{+0.014+0.026}$ & $-1.00_{-0.09-0.18}^{+0.11+0.22}$ & $-0.08_{-0.60-1.40}^{+0.50+0.86}$ & 554.317 & 33.736 \\
\hline $\mathrm{SN}+\mathrm{BAOI}+\mathrm{CMB}+\mathrm{HUB}+\mathrm{CBMF}$ & $0.293_{-0.007-0.017}^{+0.013+0.025}$ & $-1.04_{-0.10-0.21}^{+0.15+0.29}$ & $-0.50_{-0.85-1.71}^{+0.64+1.16}$ & 600.747 & 36.651 \\
\hline $\mathrm{SN}+\mathrm{BAOI}+\mathrm{CMB}+\mathrm{HUB}+\mathrm{RBAO}+\mathrm{CBMF}$ & $0.290_{-0.008-0.017}^{+0.011+0.021}$ & $-1.00_{-0.08-0.19}^{+0.16+0.28}$ & $-0.71_{-0.86-1.73}^{+0.55+1.03}$ & 602.267 & 38.815 \\
\hline
\end{tabular}

TABLE III: The best-fitted values with $68 \%$ and $95 \%$ C.L. errors of $\Omega_{m 0}, w_{0}, w_{1}$, and figure of merit for Type I combinations in the CPL model.

are GRB, RBAO, HUB and CBMF data. In what follows, we further study the properties of each data set based on basic combinations and their effects on fitting results; compare combinations of Type I and Type II; and find out the combination with the largest FoM.

\section{A. Properties of Data Sets with Two Basic Combinations}

\section{GRB}

In Table III and IV, it is obvious that the results of $\mathrm{SN}+\mathrm{BAOI}+\mathrm{CMB}+\mathrm{GRB}$ (with GRB) and $\mathrm{SN}+\mathrm{A}+\mathrm{R}+\mathrm{GRB}$ are almost the same as those of $\mathrm{SN}+\mathrm{BAOI}+\mathrm{CMB}$ and $\mathrm{SN}+\mathrm{A}+\mathrm{R}$ (without GRB), respectively. This is in accord with the results in the previous section. We have also tested other combinations that contain the GRB data, and arrived the same conclusion. As a result, we conclude that at present, adding GRB data to other observational data does not give better constraints on the DE parameters.

\section{RBAO}

This set of data is used only in Type I combinations. The inclusion of RBAO data always increases the value of the best-fitted $w_{0}$. In Table III, comparing results of $\mathrm{SN}+\mathrm{BAOI}+\mathrm{CMB}, \mathrm{SN}+\mathrm{BAOI}+\mathrm{CMB}+\mathrm{HUB}, \mathrm{SN}+\mathrm{BAOI}+\mathrm{CMB}+\mathrm{CBMF}$, $\mathrm{SN}+\mathrm{BAOI}+\mathrm{CMB}+\mathrm{HUB}+\mathrm{CBMF}$ with results of those with addition of RBAO data, we can 


\begin{tabular}{|c|c|c|c|c|c|}
\hline data & $\Omega_{m 0}$ & $w_{0}$ & $w_{1}$ & $\chi_{\text {min }}^{2}$ & FoM \\
\hline \hline $\mathrm{SN}+\mathrm{A}+\mathrm{R}$ & $0.277_{-0.015-0.028}^{+0.014+0.029}$ & $-1.01_{-0.11-0.22}^{+0.14+0.27}$ & $-0.09_{-0.66-1.47}^{+0.72+1.18}$ & 542.642 & 27.930 \\
\hline $\mathrm{SN}+\mathrm{A}+\mathrm{R}+\mathrm{GRB}$ & $0.277_{-0.014-0.027}^{+0.014+0.030}$ & $-1.01_{-0.11-0.23}^{+0.13+0.27}$ & $-0.05_{-0.69-1.51}^{+0.68+1.15}$ & 565.873 & 28.086 \\
\hline $\mathrm{SN}+\mathrm{A}+\mathrm{R}+\mathrm{CBMF}$ & $0.277_{-0.014-0.027}^{+0.015+0.029}$ & $-0.99_{-0.14-0.25}^{+0.12+0.26}$ & $-0.24_{-0.63-1.48}^{+0.84+1.35}$ & 585.775 & 27.181 \\
\hline $\mathrm{SN}+\mathrm{A}+\mathrm{R}+\mathrm{HUB}$ & $0.274_{-0.013-0.025}^{+0.014+0.029}$ & $-1.01_{-0.10-0.21}^{+0.14+0.27}$ & $0.01_{-0.73-1.53}^{+0.56+0.99}$ & 550.473 & 30.316 \\
\hline $\mathrm{SN}+\mathrm{A}+\mathrm{R}+\mathrm{HUB}+\mathrm{CBMF}$ & $0.275_{-0.013-0.025}^{+0.014+0.028}$ & $-1.02_{-0.09-0.21}^{+0.14+0.27}$ & $-0.01_{-0.78-1.58}^{+0.53+1.00}$ & 593.736 & 29.519 \\
\hline
\end{tabular}

TABLE IV: The best-fitted values with $68 \%$ and $95 \%$ C.L. errors of $\Omega_{m 0}, w_{0}, w_{1}$, and figure of merit for Type II combinations in the CPL model.

see that adding RBAO data improves FoM well with only small increase of $\chi^{2}$. Therefore it is worthwhile to contain RBAO data in the combinations to constrain DE models.

\section{HUB and CBMF}

From Table III and IV it is seen that containing HUB data can help to improve FoM, though the increase of FoM is much less than that of $\chi^{2}$ since there are 12 datapoints in the HUB data set.

For CBMF data, there are 42 datapoints. With addition of this set of data, there is a large increase of $\chi^{2}$, as seen in Table III and IV, while the FoMs for combinations with CBMF are always smaller than those without CBMF, except the case that the HUB data is also included in Type I combinations. As a result, it seems not necessary to include the CBMF data to fit DE models.

But it can be seen from Table III that based on the basic combination $\mathrm{SN}+\mathrm{BAOI}+\mathrm{CMB}$, the inclusion of both HUB and CBMF data can improve FoM well. For example, the $\mathrm{FoM}$ for $\mathrm{SN}+\mathrm{BAOI}+\mathrm{CMB}+\mathrm{HUB}+\mathrm{CBMF}$ is much larger than those for $\mathrm{SN}+\mathrm{BAOI}+\mathrm{CMB}$, $\mathrm{SN}+\mathrm{BAOI}+\mathrm{CMB}+\mathrm{HUB}$ and $\mathrm{SN}+\mathrm{BAOI}+\mathrm{CMB}+\mathrm{CBMF}$. While the data of $\mathrm{HUB}+\mathrm{CBMF}$ have no similar effect in combinations of Type II, as shown in Table IV. Therefore, in Type I combinations it had better include both HUB and CBMF data, while for Type II combinations it is not necessary to contain the CBMF data. It is also shown that with data of HUB and CBMF the best fitted $\Omega_{m 0}$ is always larger than the ones in the cases without HUB or CBMF. 


\section{B. Comparing the Two Types of Combinations}

Both basic combinations of Type I and II consist of data from Type Ia Supernovae, Baryon Acoustic Oscillation, and Cosmic Microwave Background Radiations. The FoM and $\Omega_{m 0}$ from two basic combinations $(\mathrm{SN}+\mathrm{BAOI}+\mathrm{CMB}$ and $\mathrm{SN}+\mathrm{A}+\mathrm{R})$ are very close, and $\chi_{\min }^{2}$ from $\mathrm{SN}+\mathrm{BAOI}+\mathrm{CMB}$ is a bit larger than that from $\mathrm{SN}+\mathrm{A}+\mathrm{R}$ since there is a bit more

datapoints in $\mathrm{SN}+\mathrm{BAOI}+\mathrm{CMB}$. In general, $\chi_{\min }^{2}$ in Type II are always smaller than those of the corresponding combinations in Type I. The fitting procedure of Type II combinations is much simpler than that of Type I. In Type II combinations all datapoints are only dependent on distance measurement (i.e., one needs only to use $H(z)$ ). In addition, the best-fitted $w_{0}$ $\left(\Omega_{m 0}\right)$ in Type I combinations are always smaller (larger) than the corresponding ones in Type II combinations.

\section{Recommended Combinations}

Now we take the value of FOM to be the diagnostic to quantify the ability to constrain the DE parameters for different combinations of observational data sets. From Table III and IV, we can see the combinations with the largest FoM for two types of combinations are respectively: Combination I (COM I), SN+BAOI+CMB+HUB+RBAO+CBMF for Type I, with $\mathrm{FoM}=38.815$ and Combination II (COM II), SN+A+R+HUB for Type II, with $\mathrm{FoM}=30.316$.

Though the FoM of COM II is much smaller than that of COM I, its fitting procedure is much simpler than that of COM I. The combination with the best FoM in Type I is just the one that contains the most data sets, while it is not the case in Type II combinations. The FoM for $\mathrm{SN}+\mathrm{A}+\mathrm{R}+\mathrm{HUB}+\mathrm{CBMF}$ (which owes the most sets in Type II combinations) is smaller than that for COM II (which does not contain the CBMF data). As shown in the previous section, adding CBMF data decreases FoM, and unlike the case in the Type I combinations the combination of CBMF and HUB data cannot improve FoM for Type II combinations well.

To get tight constraints of DE parameters, we therefore suggest adopting COM I to fit DE models. On the other hand, if one prefers to use less data, a simper procedure and quicker calculation, COM II would be a good choice. Of course fitting results by using COM I or 
COM II (or other combinations) will be a bit different. For the CPL model, the best-fitted $\Omega_{m 0}$ from COM I is a bit larger than that from COM II.

\section{SUMMARY}

We have analyzed the fitting properties of 11 widely-used observational data sets and their combinations. All data sets have been fitted with the CPL model, and the figure of merit (FoM) has been calculated for each case to estimate constraints on $w_{d e}$. The FoM is considered as the diagnostic to quantify the ability to constrain the DE model for different combinations of observational data sets.

We first studied the constraint properties of 11 observational data sets and their combinations. The main results are:

1. The case with the SN data used only gives a largish best-fitted $\Omega_{m 0}$, and a relative small FoM, which means that the constraints on the DE parameters are very weak in this case.

2. The addition of HUB data to SN data can help to increase FoM and the best-fitted $\Omega_{m 0}$ becomes much smaller than that from the case with SN data used only.

3. The addition of GRB data has almost no improvement in FoM, and fitting results of $\mathrm{SN}+\mathrm{GRB}$ have almost no difference from those of SN. In this sense it seems not necessary to add GRB data in order to constrain simple DE models like the CPL model.

4. The addition of $42 \mathrm{CBMF}$ seems not quite useful to constrain DE models.

5. With addition of CMB data to SN data, the FoM and constraints on the DE parameters are greatly improved than the case with SN data used only.

We also compare fitting results with several BAO data sets. With these results, we studied different combinations of observational data sets and divided them in two types, based on two widely-used basic combinations: $\mathrm{SN}+\mathrm{BAOI}+\mathrm{CMB}$ and $\mathrm{SN}+\mathrm{A}+\mathrm{R}$. We reach the following conclusions:

1. It is helpful to constrain DE models by adding the RBAO data into combinations of Type I.

2. In Type I combinations the inclusion of both HUB and CBMF data can improve FoM well, while in Type II combinations including CBMF data seems not helpful.

3. The best-fitted $w_{0}\left(\Omega_{m 0}\right)$ in Type I combinations are always smaller (larger) than the 
corresponding ones in Type II combinations.

4. To get tight constraints of DE parameters, which means a larger FoM, we suggest to use the $\mathrm{SN}+\mathrm{BAOI}+\mathrm{CMB}+\mathrm{HUB}+\mathrm{RBAO}+\mathrm{CBMF}(\mathrm{COM} \mathrm{I})$ to fit DE models. On the other hand, if one prefers less data, simper procedure and quicker calculation, the combination $\mathrm{SN}+\mathrm{A}+\mathrm{R}+\mathrm{HUB}(\mathrm{COM}$ II) is a good choice.

Obviously COM I and COM II will lead to a bit different fitting results of the cosmological parameters. For example, for the CPL model the best-fitted $\Omega_{m 0}$ of COM I is larger than that of COM II. In some cases, different combinations of data sets give rather different fitting results. This is mainly due to the fact that at present some experiment data are not accurate enough and sometimes there may exist tensions among them. Obtaining tight constraints of $w_{d e}$ and finding the nature of DE need more accurate experiments. The method of FoM used in this paper can also be applied to more new observational data[40, 60, 61] to find good combinations of data.

Acknowledgements This work was supported in part by the National Natural Science Foundation of China (No. 10821504, No. 10975168, No.11035008 and No.11075098), by the Ministry of Science and Technology of China under Grant No. 2010CB833004 and by a grant from the Chinese Academy of Sciences.

[1] A. G. Riess et al. [Supernova Search Team Collaboration], Astron. J. 116, 1009 (1998) arXiv:astro-ph/9805201.

[2] S. Perlmutter et al. [Supernova Cosmology Project Collaboration], Astrophys. J. 517, 565 (1999) arXiv:astro-ph/9812133.

[3] E. J. Copeland, M. Sami and S. Tsujikawa, Int. J. Mod. Phys. D 15, 1753 (2006) arXiv:hep-th/0603057].

[4] M. Li, X. -D. Li, S. Wang, Y. Wang, Commun. Theor. Phys. 56, 525-604 (2011). arXiv:1103.5870 [astro-ph.CO]].

[5] R. R. Caldwell and P. J. Steinhardt, Phys. Rev. D 57, 6057 (1998) arXiv:astro-ph/9710062.

[6] P. J. Steinhardt, L. M. Wang and I. Zlatev, Phys. Rev. D 59, 123504 (1999) arXiv:astro-ph/9812313.

[7] S. Capozziello, S. Carloni and A. Troisi, Recent Res. Dev. Astron. Astrophys. 1, 625 (2003) 
arXiv:astro-ph/0303041].

[8] R. G. Cai, Phys. Lett. B 657, 228 (2007) [arXiv:0707.4049 [hep-th]];

[9] H. Wei and R. G. Cai, Phys. Lett. B 660, 113 (2008) [arXiv:0708.0884 [astro-ph]].

[10] R. G. Cai and Q. Su, Phys. Rev. D 81, 103514 (2010) arXiv:0912.1943 [astro-ph.CO]].

[11] B. Feng, X. L. Wang and X. M. Zhang, Phys. Lett. B 607, 35 (2005) arXiv:astro-ph/0404224].

[12] M. X. Luo and Q. P. Su, Phys. Lett. B 626, 7 (2005) arXiv:astro-ph/0506093.

[13] C. Gao, X. Chen and Y. G. Shen, Phys. Rev. D 79, 043511 (2009) [arXiv:0712.1394[astro-ph]].

[14] R. G. Cai, Q. Su, Z. L. Tuo and H. B. Zhang, arXiv:1011.3212 [astro-ph.CO].

[15] M. Chevallier and D. Polarski, Int. J. Mod. Phys. D 10, 213 (2001) [arXiv:gr-qc/0009008.

[16] E. V. Linder, Phys. Rev. Lett. 90, 091301 (2003) arXiv:astro-ph/0208512].

[17] D. Huterer and M. S. Turner, Phys. Rev. D 64, 123527 (2001) arXiv:astro-ph/0012510].

[18] A. R. Cooray and D. Huterer, Astrophys. J. 513, L95 (1999) arXiv:astro-ph/9901097.

[19] D. Huterer and M. S. Turner, Phys. Rev. D 60, 081301 (1999) arXiv:astro-ph/9808133.

[20] J. Weller and A. J. Albrecht, Phys. Rev. Lett. 86, 1939 (2001) arXiv:astro-ph/0008314.

[21] D. Huterer and G. Starkman, Phys. Rev. Lett. 90, 031301 (2003) arXiv:astro-ph/0207517.

[22] D. Huterer and A. Cooray, Phys. Rev. D 71, 023506 (2005) arXiv:astro-ph/0404062.

[23] A. Hojjati, L. Pogosian and G. B. Zhao, JCAP 1004, 007 (2010) arXiv:0912.4843 [astroph.CO]].

[24] Y. Wang, Phys. Rev. D 80, 123525 (2009) [arXiv:0910.2492 [astro-ph.CO]].

[25] T. Holsclaw, U. Alam, B. Sanso, H. Lee, K. Heitmann, S. Habib, D. Higdon, Phys. Rev. Lett. 105, 241302 (2010) arXiv:1011.3079 [astro-ph.CO]].

[26] T. Holsclaw, U. Alam, B. Sanso, H. Lee, K. Heitmann, S. Habib, D. Higdon, Phys. Rev. D82, 103502 (2010) [arXiv:1009.5443 [astro-ph.CO]].

[27] T. Holsclaw, U. Alam, B. Sanso, H. Lee, K. Heitmann, S. Habib, D. Higdon, arXiv:1104.2041 [astro-ph.CO]].

[28] S. Sullivan, A. Cooray and D. E. Holz, JCAP 0709, 004 (2007) arXiv:0706.3730 [astro-ph]].

[29] R. G. Cai, Q. Su and H. B. Zhang, JCAP 1004, 012 (2010) arXiv:1001.2207 [astro-ph.CO]].

[30] G. B. Zhao, D. Huterer and X. Zhang, Phys. Rev. D 77, 121302 (2008) arXiv:0712.2277 [astro-ph]].

[31] P. Serra, A. Cooray, D. E. Holz, A. Melchiorri, S. Pandolfi and D. Sarkar, Phys. Rev. D 80, 121302 (2009) [arXiv:0908.3186 [astro-ph.CO]]. 
[32] S. Nesseris and L. Perivolaropoulos, JCAP 0702, 025 (2007) arXiv:astro-ph/0612653.

[33] J. C. B. Sanchez, S. Nesseris and L. Perivolaropoulos, JCAP 0911, 029 (2009) [arXiv:0908.2636 [astro-ph.CO]].

[34] H. Wei, Phys. Lett. B 687, 286 (2010) arXiv:0906.0828 [astro-ph.CO]].

[35] Y. Gong, B. Wang and R. g. Cai, JCAP 1004, 019 (2010) arXiv:1001.0807 [astro-ph.CO]].

[36] Z. Li, P. Wu and H. Yu, Phys. Lett. B 695, 1 (2011) arXiv:1011.1982 [gr-qc]].

[37] R. Lazkoz, S. Nesseris, L. Perivolaropoulos, JCAP 0807, 012 (2008) arXiv:0712.1232 [astro$\mathrm{ph}]]$.

[38] C. Escamilla-Rivera, R. Lazkoz, V. Salzano, I. Sendra, JCAP 1109, 003 (2011) arXiv:1103.2386 [astro-ph.CO]].

[39] I. Maor, R. Brustein and P. J. Steinhardt, Phys. Rev. Lett. 86, 6 (2001) [Erratum-ibid. 87, 049901 (2001)] arXiv:astro-ph/0007297.

[40] A. Albrecht et al., arXiv:astro-ph/0609591.

[41] J. Dossett, J. Moldenhauer, M. Ishak, Phys. Rev. D84, 023012 (2011) arXiv:1103.1195 [astroph.CO]].

[42] I. Sendra, R. Lazkoz, N. Benitez, arXiv:1105.4943 [astro-ph.CO]].

[43] R. Amanullah et al., Astrophys. J. 716, 712 (2010) [arXiv:1004.1711 [astro-ph.CO]].

[44] E. Komatsu et al. [WMAP Collaboration], Astrophys. J. Suppl. 180, 330 (2009) arXiv:0803.0547 [astro-ph]].

[45] S. Burles, K. M. Nollett and M. S. Turner, Astrophys. J. 552, L1 (2001) arXiv:astro-ph/0010171].

[46] Y. Wang, Phys. Rev. D 77, 123525 (2008) [arXiv:0803.4295 [astro-ph]].

[47] H. Wei, JCAP 1008, 020 (2010) arXiv:1004.4951 [astro-ph.CO]].

[48] N. Liang, W. K. Xiao, Y. Liu and S. N. Zhang, arXiv:0802.4262 [astro-ph].

[49] S. W. Allen, D. A. Rapetti, R. W. Schmidt, H. Ebeling, G. Morris and A. C. Fabian, Mon. Not. Roy. Astron. Soc. 383, 879 (2008) [arXiv:0706.0033 [astro-ph]].

[50] L. Xu and J. Lu, JCAP 1003, 025 (2010) arXiv:1004.3344 [astro-ph.CO]].

[51] S. Nesseris and L. Perivolaropoulos, JCAP 0701, 018 (2007) arXiv:astro-ph/0610092.

[52] D. Stern, R. Jimenez, L. Verde, M. Kamionkowski and S. A. Stanford, JCAP 1002, 008 (2010) arXiv:0907.3149 [astro-ph.CO]].

[53] A. G. Riess et al., Astrophys. J. 699, 539 (2009) [arXiv:0905.0695 [astro-ph.CO]]. 
[54] E. Komatsu et al. [WMAP Collaboration], Astrophys. J. Suppl. 192, 18 (2011) arXiv:1001.4538 [astro-ph.CO]].

[55] W. Hu and N. Sugiyama, Astrophys. J. 471, 542 (1996) arXiv:astro-ph/9510117.

[56] B. A. Reid et al. [SDSS Collaboration], Mon. Not. Roy. Astron. Soc. 401, 2148 (2010) arXiv:0907.1660 [astro-ph.CO]].

[57] D. J. Eisenstein and W. Hu, Astrophys. J. 496, 605 (1998) arXiv:astro-ph/9709112.

[58] D. J. Eisenstein et al. [SDSS Collaboration], Astrophys. J. 633, 560 (2005) arXiv:astro-ph/0501171].

[59] E. Gaztanaga, R. Miquel and E. Sanchez, Phys. Rev. Lett. 103, 091302 (2009) arXiv:0808.1921 [astro-ph]].

[60] A. Conley, J. Guy, M. Sullivan, N. Regnault, P. Astier, C. Balland, S. Basa, R. G. Carlberg et al., Astrophys. J. Suppl. 192, 1 (2011). arXiv:1104.1443 [astro-ph.CO]].

[61] M. Sullivan, J. Guy, A. Conley, N. Regnault, P. Astier, C. Balland, S. Basa, R. G. Carlberg et al., Astrophys. J. 737, 102 (2011). [arXiv:1104.1444 [astro-ph.CO]]. 